\title{
How Much of the FUTURE Is BEHIND in Arabic? A View on the Arabic Culture and Embodiment
}

\author{
Maisarah M. Almirabi \\ Department of English, Umm Al-Qura University, Makkah, Saudi Arabia
}

\begin{abstract}
In the present study, the Arabic metaphorical expressions associated with the conceptual metaphors TIME IS SPACE and THE FUTURE IS BEHIND were analyzed. The analyzed tokens were searched for online. In addition, native speakers of Hijazi-Saudi Arabic confirmed the natural usage of the tokens in their dialect. The productivity of placing the FUTURE in front of the EGO and the unproductivity of placing it behind indicates the FUTURE in front of the EGO as the norm. Based on the metaphorical elements found in the tokens considered, a bidimensional conceptual location of objects on the JOURNEY OF TIME was proposed to include the front location or the elsewhere location. The elsewhere location was referred to as behind, extending the meaning of 'behind' to include all locations that are not front. This bidimensionality is represented by the conceptual metaphors FOCUS IS FRONT and PERIPHERY IS BEHIND. Examples associated with these conceptual metaphors were associated with the experiential embodiment. In previous research, the direction of writing and how much weight is given to cultural values have claimed to influence the placement of the FUTURE in Arabic as pre-set reasons. This study is significant because it is done without pre-set reasons for metaphor usage, resulting in none-steered findings. Also, this study opens a window to the metaphorical system of the Hijazi-Saudi Arabic, a variety of Arabic whose metaphorical system is understudied. This study invites considering the placement of the FUTURE in other languages and cultures.
\end{abstract}

Index Terms - metaphors, conceptual metaphor theory, cognitive linguistics, Hijazi Saudi, Arabic, future is behind

\section{INTRODUCTION}

A language is a system for communicating and sharing ideas. Some of these ideas are abstract and do not have readily available names, nor are they related to conceptual structures. This necessitates language users to find alternative references and conceptual structures to communicate about such abstract entities. Lakoff and Johnson (2003) listed three metaphor functions: ontological, structural, and orientational. When the metaphor concretizes abstract concepts, it has an ontological function. When it gives a complex structure to the concept, it has a structural function. And when the metaphor contributes to the coherence of some concepts, it has an orientational function. The ontological function is in effect when providing a reference to TIME, as in sentences 1 and 2.

1. Thank you for your time.

2. Can you spare a minute?

Notice how the concepts of TIME are concretized as a possession and a commodity in examples 1 and 2, respectively. Such ontological metaphors allow us to talk about the abstract concept of TIME, which cannot otherwise be discussed due to its abstract nature. Sentences 3 and 4 are examples of structural metaphors.

3. A bright future is waiting for you. (Indicating the result of hard work, for example)

4. We need to go our separate ways. (In the context of ending a personal relationship)

The metaphors LIFE IS A JOURNEY and LOVE IS A JOURNEY are examples of mapping the structure of one thing or entity to another. Consider examples 3 and 4. The conceptual metaphor LIFE IS A JOURNEY demonstrates how living is like traveling, as the PAST, PRESENT, and FUTURE are passed by, situated at, or targeted stations, respectively.

Arabic time terms represent fossilized conceptualization of the metaphor LIFE IS A JOURNEY. The PAST, PRESENT, and FUTURE expressions in Arabic literally mean passed by, situated at, and headed toward (station or direction), respectively. The root of the word for past $/ \mathrm{maDi} /$ means "passed thing or person". The word for the present /HaDir/ means "attendee", while the word for future, /mustaqbal/, means "headed toward".

Life is explained as a continuum consisting of three events: the past, present, and future (Meriam-Webster Dictionary, retrieved 10/7/19). This continuum is logically parallel to the conceptualized JOURNEY of LIFE. Life is the period of existence; hence, it is a time experienced individually by someone. The fact that humans experience life makes it a fertile ground for experiential embodiment.

According to Lakoff (1993), the passage of time within the JOURNEY metaphor can be conceptualized in two ways. Time passing is concretized as motion within the conceptual metaphor TIME PASSING IS MOTION. As Lakoff (1993) 
described, this conceptual metaphor has two special cases: TIME PASSING IS MOTION OF AN OBJECT and TIME PASSING IS MOTION OVER A LANDSCAPE. Lakoff (1993) provides the example "time is flying by" for the conceptual metaphor TIME IS MOTION OF AN OBJECT, and the example "I'll be there in a minute" for the conceptual metaphor TIME PASSING IS MOTION OVER A LANDSCAPE (Lakoff 1993, p. 14). Linking the attribute of flying to TIME indicates TIME itself is a moving object. On the other hand, implying that a point in time is where one is situated concretizes time as a landscape in which locations are points in time. These ways of concretizing time conceptualize the direction the EGO is heading toward is in front and not behind. This is one way of conceptualizing time in language.

Another version of direction in the JOURNEY OF LIFE was found to exist. Lakoff and Johnson (2003) explained some apparent contradictions in the organization of front-back metaphors. They provided examples such as "We're looking ahead to the following weeks" (Lakoff \& Johnson, 2003, p. 42). In this example, the FUTURE is in front of, ahead, and behind, following the speaker. The authors explained that there were two types of front-back orientation, namely motion-sourced and canonical. Motion-sourced orientation is acquired by objects due to their motion. This is based on the front and back of humans, animals, and vehicles, as motion occurs when the front of the human or animal body or vehicle is heading forward. However, the canonical orientation is related to the existing or designated front and back of the human, animal, or vehicle. This means that a reversing car retains its canonical front, although the motionsourced orientation while reversing is the opposite (Lakoff \& Johnson, 2003). An example of motion-sourced orientation is a rock thrown through the air, which then hits and breaks a windshield. To explain the windshieldbreaking incident, we may describe that as "the rock has a pointy front, which caused a bullseye break in the windshield", for example. Notice how this front is based on the motion of the rock.

The peculiarity of human bodies has been a significant influence on the generation of conceptual metaphors (Lakoff and Johnson, 1999). Núñez (1999) used the TIME IS SPACE metaphor to support the idea that our brains and bodily peculiarities are important in structuring conceptual metaphors. Núñez claimed that bodily orientation and actions are factors in making the concept of 'time flow' possible. Two types of embodiment play significant roles in producing time metaphors which are.

- The experiential embodiment (to indicate how the repeated experience produces a conceptual structure that can be lent to less concretized concepts).

- The ego presence/locus embodiment (to indicate the location of concepts relative to the location of the ego).

The connection between these types of embodiment and time metaphors will be explained later in the present study.

The current study answers the following questions:

1. How do Arabic speakers concretize TIME concepts?

2. Which of the embodiment types are used in Arabic?

3. How culturally specific are time expressions in Arabic?

\section{LITERATURE REVIEW}

The discussion of the conceptualization of time has a long history. Time has been claimed to be unreal as, linguistically, it involves talking about something that is not time per se, such as space as a source domain (McTaggart, 1908). More recently, spatial experience has been claimed to influence temporal conceptualizations (e.g., Boroditsky, 2000; Boroditsky \& Ramscar, 2002; Gentner et al., 2002; Kranjec, 2006; Núnez et al., 2006). In different cultures around the world, time was proposed to be conceptually represented in different ways. People of Pormpuraaw, an Australian aboriginal community, conceptualize time as flowing toward the position of the EGO using an orientation that is relative to the movement of the sun. In this conceptual structure, the direction toward which the EGO is facing determines the direction of TIME. The TIME approaches from the right, the left, toward, and away, corresponding to the EGO facing north, south, east, and west, respectively. The Pormpuraaw's structuring of time is a result of experiential embodiment in which the daily experience of the movement of the sun plays an interesting role in the production of the metaphor TIME IS SPACE.

In another study, an allocentric conception of time was found in a socially isolated group living in the Yupno valley of Papua New Guinea. Members of this group pointed uphill to indicate the FUTURE and downhill to indicate the PAST (Núñez et al., 2012). The metaphorical motivation for the speakers in the Yupno valley was environmentally based, as the group structured TIME based on the vertical orientation relative to a hill (uphill/downhill) (Núñez et al., 2012). The speakers of Yupno conceptualized a world that was historically associated with their native place of settlement. This world was associated with their homes (referring to land and building). When away from their homeland, they could only use the vertical orientation inside their home buildings, reflecting the association between the building and land based on the HOME concept (Núñez et al., 2012). This implies a metonymy of location in which the homeland is represented by a "home" building. Natural surroundings such as the sun and a hill can be used to form a conceptual structure. Using the sun is available for every culture since every human being, in normal and common situations, experiences the sun. However, using a certain hill is only available for the people who have been experiencing the hill. Generally, different landmarks are experienced in different cultures worldwide, which make them available to be utilized in the respective cultures. Cultural specificity will be further discussed in association with the tokens analyzed in the present study. 
De la Fuente et al. (2014) proposed the temporal focus hypothesis (TFH), which brings the mentally focused things in front. They applied this hypothesis to Moroccan Arabic and Spanish participants. They found that Moroccans focused more on past events than all participating Spaniards. They also found that elder Spaniards focused more on past events than young Spaniards. They claim that focusing more on past events indicates giving more importance to past events (traditions) than future events (progress). Callizo-Romero et al. (2020) replicated the test of the TFH on more groups of participants that represent larger diversity. Similar to De la Fuente et al. (2014), they concluded that past events could either attract or distract focus depending on how participants regard the implications of these events. In addition, personal and societal attitudes were concluded to project onto the time of focus. These studies propose some interesting results; however, their methodology did not give sufficient space to explore other reasons for temporal focus. Reasons for temporal focus are expected to be more abundant in naturally and spontaneous mental products such as language and gestures.

The direction of writing was proposed to affect the conceptualization of time. More specifically, the flow of events within the TIME IS SPACE metaphor was found to be associated with the direction of writing. The flow of event direction matched the direction of writing when testing speakers of Arabic, Hebrew, English, Spanish, and Italian (Chatterjee, Maher, Gonzalez-Rothi, \& Heilman, 1995; Maher, Chatterjee, Gonzalez-Rothi, \& Heilman, 1995, Santiago et al. 2007, Maas \& Russo, 2003, and others). English and Mandarin speakers use front/back and up/down metaphors to refer to time, but Mandarin speakers are more likely to think about time vertically than are English speakers (Boroditsky, 2001; Boroditsky, Fuhrman, \& McCormick, 2011). Although matching the direction of writing with recognizing the flow of temporal events produces interesting results, it is based on unnatural stimuli. It is important to mention that excluding natural representations of mental processes such as language and gestures disregards time spatialization patterns, including where to locate the FUTURE and on what axis. In addition, such a test limits the scope of the investigation to the effect of frequently experienced patterns of writing direction only.

Finding expressions that don't reflect the direction of writing cannot be accounted for without analyzing natural data and matching analysis findings to context. In the present study, reasons for situating TIME in relevance to the EGO were proposed based on what language users choose to use before collecting data in naturally produced linguistic elements. The present study focused on the Hijazi-Saudi Arabic, and the analyzed tokens were linked to the cultural context of its speakers.

The FUTURE is conceptualized as being behind the EGO utilizing the conceptual metaphor UNKOWN IS BEHIND (Dahl, 1995, Núñez \& Sweetser, 2006, Sullivan \& Bui, 2016). Dahl (1995) stated that Malagasy speakers attribute this to the fact that the FUTURE is unknown in connection with the concept that what is not seen is unknown. This is logically explained by not having eyes in the back of our human heads; hence, we cannot see what is behind us. On the other hand, Malagasy speakers conceptualize the past and present as being in front of them since both the past and the present are known, and what is known should be visible. Dahl (1995, p. 199) also claimed that a sentence such as "arahaba fa tratry nytaona (congratulations, for being reached by the year)" was evidence that people did not reach the year, but that the year approached the person from behind. This concept is similar to Lakoff's (1993) conceptual metaphor TIME PASSING IS MOTION OF AN OBJECT, in which the moving object is the year. Dahl (1995) proposed that Malagasy speakers use three ways to conceptualize time. Time is conceptualized as linear, cyclical, and event-related. The linear TIME is future-oriented, the cyclical is past-oriented, and the event-related is present-oriented. In the present study, it is proposed that time is linear as travelers commute on a timeline. However, different events along the way interfere with this travel progress and impose unplanned changes to the trip. This will be picked up later when considering the turning around of TRAVELERS to face other TRAVELERS on their respective JOURNEYS. Those journeys happen to share the same conceptual trajectory or crossing point of trajectories.

The FUTURE is also conceptualized behind in Aymara and Vietnamese cultures based on the same concept - that it is unknown, and what is unknown must be behind us (Núñez and Sweetser 2006, Sullivan and Bui 2016). Núñez and Sweetser (2006) explained that metaphorical gestures should be coupled with speech gestures to obtain the full picture of the placement of the EGO within the conceptual metaphor. When analyzing metaphoric gestures, Sullivan and Bui (2016) found that Vietnamese speakers conceptualized the FUTURE as approaching from behind, passing them, becoming the past, and continuing. Although considering gestures in analyzing time metaphors is important, they will not be considered since the data analyzed was produced before the study, resulting in a lack thereof.

\section{MethodOLOGY}

Of the results, the first 100 tokens of those originally posted and not intended for research were considered.

In the current study, the author collected data from online sources via the Google search engine. This domain contains naturally occurring discourse not usually constructed for research purposes (see Silverman, 2013). Key expressions that represent the TIME IS A JOURNEY metaphor were searched for. These expressions include the Arabic word that refers to the future 'مستقبل' together with an Arabic action verb indicating a TRIP movement, such as

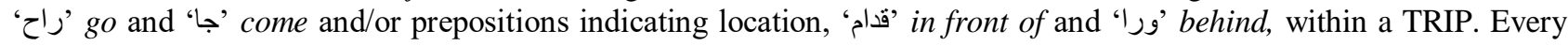
search resulted in a minimum of 168 results. The first 100 results of each key expression posted for non-research purposes were analyzed using the Metaphor Identification Procedure (MIP), described in the next section. Considering 
only the first 100 tokens puts a threshold on the number of tokens, as some searches resulted in several hundred results. The genre of the tokens was determined via the linguistic and website contexts.

The MIP goes as following:

1. Read the entire text-discourse to establish a general understanding of the meaning.

2. Determine the lexical units in the text-discourse

3. (a) For each lexical unit in the text, establish its meaning in context, that is, how it applies to an entity, relation, or attribute in the situation evoked by the text (contextual meaning). Take into account what comes before and after the lexical unit.

(b) For each lexical unit, determine if it has a more basic contemporary meaning in other contexts than the one in the given context. For our purposes, basic meanings tend to be

-More concrete; what they evoke is easier to imagine, see, hear, feel, smell, and taste.

- Related to bodily action.

- More precise (as opposed to vague)

- Historically older.

Basic meanings are not necessarily the most frequent meanings of the lexical unit.

(c) If the lexical unit has a more basic current-contemporary meaning in other contexts than the given context, decide whether the contextual meaning contrasts with the basic meaning but can be understood in comparison with it.

4. If yes, mark the lexical unit as metaphorical. (Pragglejaz Group 2007, p. 3)

The embodiment and cultural specificity of metaphors and metaphorical patterns were also considered, and conceptual associations were proposed.

Only one example of each key expression was listed below. The transcription convention used in transcribing Arabic examples was provided in the appendix.

When a word or expression referred to a concept, all letters were capitalized.

\section{ANALYSIS}

TIME is conceptualized as having the structure of a JOURNEY in Arabic. This JOURNEY includes a TRAVELER and some other people not included in this JOURNEY. Consider examples 5 and 6:

5. رايح اكلم أمي بكره

$\begin{array}{lccc}\text { raijh } & \text { akal:im } & \text { um:i } & \text { bujkrah } \\ \text { going-1stSG } & \text { talk } & \text { mother } & \text { tomorrow }\end{array}$

I will talk to my mother tomorrow.

6. كان رايح يقولك لكن بطل

kanraijh jigul:ak lakin baT:al

was going- $2{ }^{\text {nd }}$ SGtell-INT- $-{ }^{\text {nd }} S G$ but changed mind $-3{ }^{\text {rd }}$ SG

He was planning to tell you but changed his mind.

7. رايح ارسل له الرسالة لمن أفضىى

raijh arsil lah ar:isalah lam:an afDa

going- $1^{\text {st }} \mathrm{SG}$ send to- $3^{\text {rd }} \mathrm{SG}$ DEF-message when free- $1^{\text {st }} \mathrm{SG}$

I will send the message when I am free.

8. رايح اذكرك انك غلطان

raijh aðak:irak in:ak salTan

going- $1{ }^{\text {st }} \mathrm{SG} \quad 1^{\text {st }} \mathrm{SG}$-remind-TR- $2{ }^{\text {nd }} \mathrm{SG}$ that $-2{ }^{\text {nd }} \mathrm{SG}$ wrong- $2{ }^{\text {nd }} \mathrm{SG}$

I will remind you that you were wrong.

Examples 5 to 8 view the EGO as traveling on a JOURNEY OF LIFE by including the word رايح [raj:h] "going", which literally means "going from one physical point to another". However, in example 5, a conversation is conducted telephonically; the act of going is used to imply the figurative sense of going on a JOURNEY in time. Similarly, examples 6, 7, and 8 include the metaphorical GOING into the FUTURE to perform the actions of telling, sending a message, and reminding, respectively. Notice that there is no need for physically going on a JOURNEY when planning to say something, send a message, or remind someone about something. Although examples 5 through 8 do not explicitly connote going from one location to another, going to another location could be part of the action. This is particularly the case when the person with whom one interacts is not close or there are no portable means of communication. However, other verbs do not imply a physical commute to perform the actions, as they are accomplished mentally. Consider examples 9 and 10:

9. رايح افكر في الموضوع

raijh afak:ir

going- ${ }^{\text {st }} \mathrm{SG}$ think-1 ${ }^{\text {st }} \mathrm{SG}$ in DEF-subject

I will think about this.

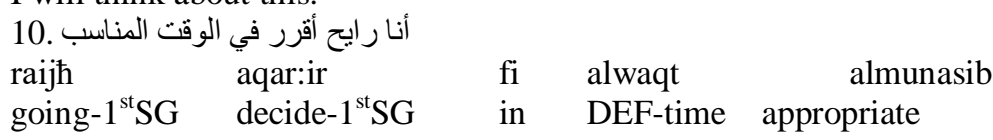


I will decide at the right time.

Notice how thinking and deciding are usually done mentally without traveling physically. In examples 9 and 10, the person attempting to think and decide expresses this via the metaphorical use of "going", although they are not physically going into the FUTURE, but doing so conceptually in the JOURNEY OF LIFE.

Note the similarity to the English modal verb 'be going to' concerning going into the FUTURE. Consider examples 11-14, taken from the Corpus of Contemporary American English (COCA).

11. I am going to consider my options later.

12. I'm going to prove to you that I can make it.

13. I am going to show them they're wrong.

14. I think she's actually going to miss you.

In examples 11-14, considering, proving, showing, and missing do not require physical travel. This "going", as indicated by the function of the modal verb, is into the FUTURE, which indicates the metaphorical concept of the LIFE IS A JOURNEY metaphor. Conceptualizing LIFE or TIME as a concrete concept such as a JOURNEY allows for talking about LIFE or TIME, although the intangibility due to their abstract nature disallows talking about them otherwise. The conceptual structure of a JOURNEY is projected onto the abstract concepts of TIME and LIFE. This allows the use of distance, orientation, and TRAVELERS as properties of TIME and LIFE within this JOURNEY.

The orientation of the JOURNEY OF LIFE in Arabic is in front of the EGO. Consider examples 15 and 16:
15. الو عد قدام والحي بيشوف
alwaS qed:am
DEF-promise ahead
walhaj
and-DEF-alive
bifuf

The promise is ahead, and the living ones will see.

16. استمري قدامك مستقبل باهر

istamir:i qud:amik

continue- $1^{\text {st }}$ SG-FEM in front of- $1^{\text {st }}$ SG-FEM future-IN amazing

Keep on doing this. A bright future is in front of you.

Examples 15 and 16 view the first person as traveling toward the FUTURE, which is a direction in front of them, by using the word قدامنا [qud:amana] 'in front of-1 ${ }^{\text {st }} \mathrm{PL}$ ', which indicates the physical frontwards orientation. This was found productive because the FUTURE concept (example 16) and anything related to, planned for, or going to happen in the future (example 15) can be located in front of the EGO.

Expressing the FUTURE is behind the Ego is found in Arabic. Consider examples 17-19:

17. تعليق ايش انت وهو وهي يلا ع الدوام ورانا مستقبل

$\begin{array}{lllll}\text { taSliq } & \text { e; inta } & \text { wuhuwa } & \text { wehija } & \text { jal:a } \\ \text { suspension } & \text { what you-SG-MASC and-he } & \text { and-she } & \text { come on } \\ \text { Ya ad:awam } & \text { warana } & \text { mustaqbal } & & \\ \text { on work } & \text { behind-1 }{ }^{\text {st }} \mathrm{PL} \quad \text { future } & & \end{array}$

What kind of suspension are you talking about? Off you go to work, you guys.

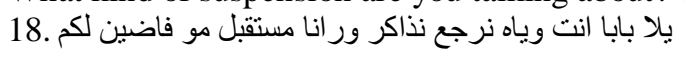

jal:a baba inta wijah nirzaS-1 ${ }^{\text {st } P L \text { niðakir }}$

Come on daddyyou-SG-MASC and-him we go back study

warana mustaqbel mu faDin lakum

behind-1 ${ }^{\text {st }} \mathrm{PL}$ future not free for you

Let's go back to studying, dudes, you and him. Our future awaits, so we are not free.

19. بنات هيا بنا نترك الدراسة ، ور انا مستقبل و وعيال

banat hija bina nitrukad:irasa warana mustaqbal

girls-VOC let's leave studying behind- ${ }^{\text {st }}$ future

weYjal

and-children (to bear and rear)

You girls, let's drop out of school. We are responsible for our future and our future children.

Example 17 shows the FUTURE as being behind people who were wondering if their jobs would be suspended due to claims of bad weather conditions. Conceptualizing the FUTURE work as being behind us indicates "work" is the means of achieving future responsibility. Example 18 describes a group of people stopping to rest while on a JOURNEY of study and heading into the FUTURE. This group probably met another group while resting, as indicated by the phrase مو فاضين لكم [mu faDin lakum] "(we are) not free from you(r nonsense)". This "stop" distracts from the ongoing JOURNEY in which the direction is the future goal, indicated by the phrase نرجع نذاكر [nirzaS-1 go back studying". In addition, STUDYING is construed as the process of attaining the goal of this JOURNEY, namely the FUTURE. Example 19 describes a group of girls embarking on a JOURNEY "of studying" and suggests leaving this path and embarking on another "of raising kids". This is the (new) way of heading toward the FUTURE, with new responsibilities and different future results, thus switching between worlds in Wierzbicka's (1973) terms ${ }^{1}$. This

\footnotetext{
${ }^{1}$ The term "trip" is used to express the concept of 'worlds' introduced by Wierzbicka (1973) to emphasize the specific metaphor TIME IS SPACE.
} 
conceptual "trip switch" is also evidence of the many conceptual TRIPS in the JOURNEY OF LIFE. The future destination, which involves raising children, is behind the EGO when expressing what needs to be done when conversing with people in the second person. Thus, examples 17-19 indicate TRAVELERS stopping to express their feelings about heading toward the FUTURE and then returning to proceed along the JOURNEY. This TRIP is construed as a responsibility. However, due to being distracted from the future responsibilities by conceptually turning around and talking to people who are not embarking on this specific TRIP, the FUTURE becomes behind the EGO due to this turning around. This is a distracting orientation. Notice how the future-result responsibility is located in the past/posterior position due to the distractions in these examples.

There is an orientational complexity in Arabic, referred to as parallel TRIPS on the JOURNEY OF LIFE, similar to the WORLDS concept introduced by Wierzbicka (1973). The "trip" and "journey" terms indicate the TRIP being part of the JOURNEY. On one of the TRIPS, the EGO and TIME are TRAVELERS. This is in line with the concepts of front-back orientations being canonical when the traveler is the EGO, or motion-sourced when the traveler is TIME (Lakoff \& Johnson, 2003). In the TRIP in which the EGO is the traveler, the EGO goes forward while facing the direction of the FUTURE; reconsider examples 5-10. This supports the idea that, while on the TRIP, TIME is moving toward the EGO when TIME is the traveler. Consider examples 20 and 21:

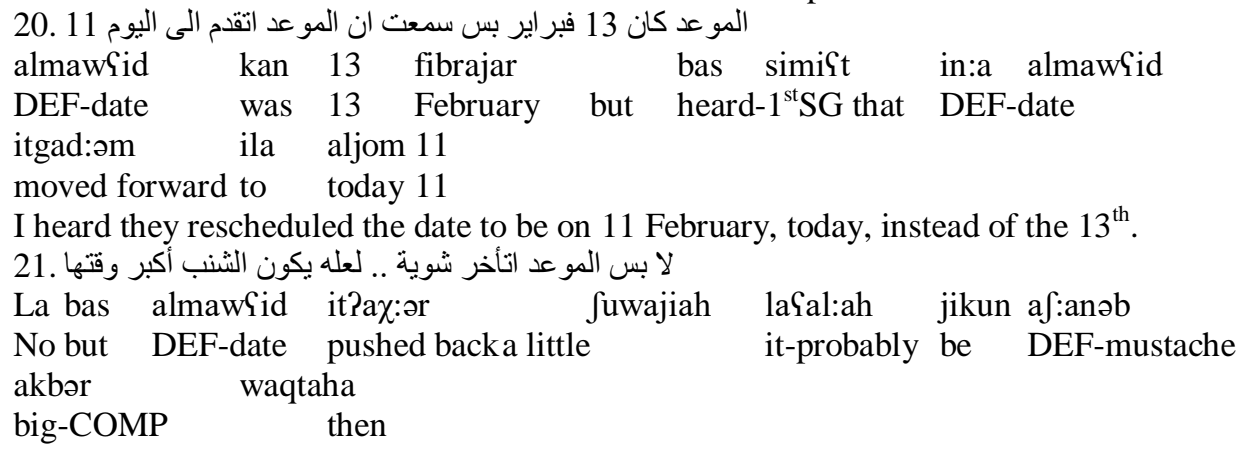

No, but the date was pushed back a little bit. This is good since the mustache will be bigger then.

The canonical orientation in which the EGO is the traveler is already conceptually implicit in the normal situation of the JOURNEY metaphor in the mind of the speaker. The other orientation, in which TIME is the traveler, is denoted by

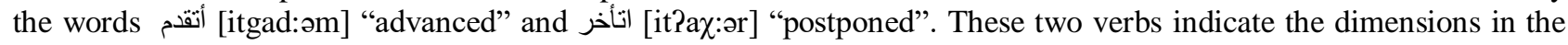
JOURNEY trajectory along which TIME can travel, that is, forwards and backward. Referring to a previous date and the growth of the mustache implies these dimensions in Examples 20 and 21, respectively. Lakoff and Johnson's (2003, p. 42) example, "We're looking ahead to the following weeks", shows the FUTURE in a similar way to Examples 20 and 21. Setting a conceptual scene helps conceptualize the FUTURE WEEKS as "following". In this scenario, the TIME represented by the FUTURE WEEKS is heading toward the EGO. This indicates that the EGO and TIME are traveling on separate TRIPS heading toward opposing directions. Consider Figure 1:

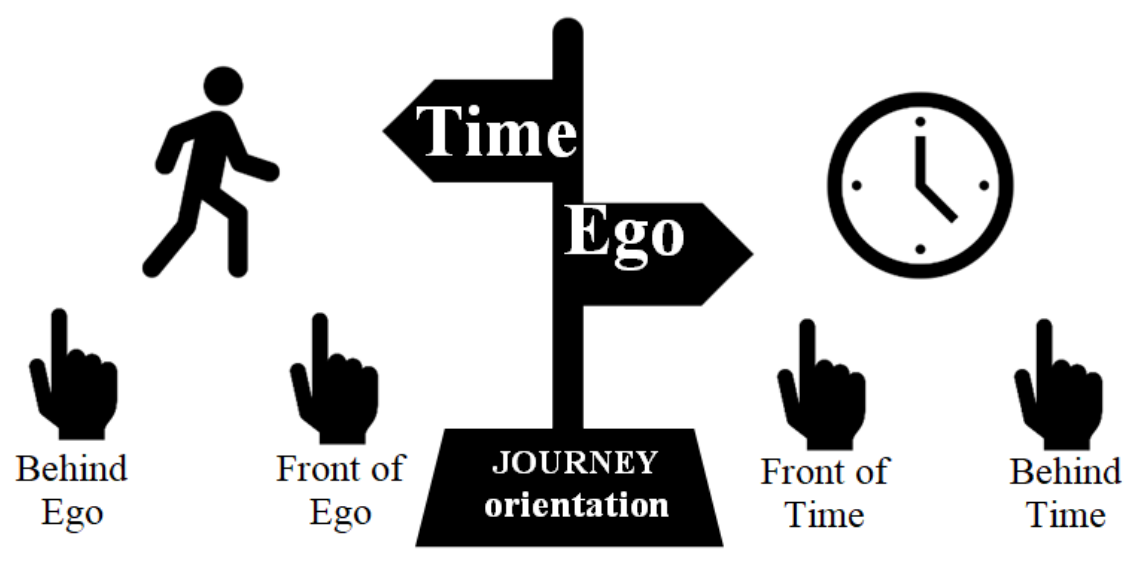

Diagram (1)

Notice how the concept of TIME IS A TRAVELER ON A JOURNEY embodies time as being a traveling human, albeit with a motion-sourced fore and aft. This indicates the partial projection of human features onto time. 
Different cultures around the world utilize different conceptual structures to concretize time-related concepts. The sun, a natural monument, and the direction of writing are examples of source domains affecting conceptualizing timerelated concepts (Dahl, 1995; Núñez \& Sweetser, 2006; Sullivan \& Bui, 2016).

Aymara, Malagasy, and Vietnamese people were found to use the behind-EGO location of the FUTURE concept. The metaphor KNOWING IS SEEING was claimed to be the source of structuring the FUTURE as behind, since the FUTURE is unknown, we see known things, and human eyes only view what is in front.

In the present study, different types of embodiment were found associated with time metaphors used by speakers of Hijazi-Saudi Arabic. In association with the metaphor, TIME IS SPACE, the experiential and ego presence/locus embodiment types were found. Metaphorical embodiment is at work when the conceptual embodiment results from experiencing a physical location and when the EGO is conceptually located within the space of time. It is logical that embodiment is universally available when not associated with culturally specific concepts, such as being in a specific culture or region. An example of this is when embodiment results from routine experiences or a general location in relation to common objects or concepts. A more specific example is the conceptual movement from inactive to active mental states. This is represented by a derivative of "going" when no physical going is taking place. This universality emanated from the relationship of these concepts to the peculiarities of the human mental experience to which all humans have access.

With regard to the FUTURE IS BEHIND metaphor, while traveling along the JOURNEY OF TIME, it is common that the FUTURE is in front of us. However, when talking to other people, we cannot help being distracted from the direction of the JOURNEY and turn around to face our interlocutors instead of the FUTURE. The conversation explains something about the journey destination, which has now become behind us due to this turning. Turning can be associated with our human body's peculiarities, which makes the turning maneuver possible, and the experiential embodiment since we tend to turn to focus on what attracts our attention. These different structural parts of the metaphor, including other TRAVELERS and the turning to face them, are the metaphorical relational and multidimensional status discussed by Gentner et al. (2002) and Feist and Duffy (2020).

Arabic metaphors placing the FUTURE in FRONT OF EGO, such as examples 15 and 16, were abundant in Arabic. However, a few Arabic metaphors were found to place the FUTURE behind EGO. Two metaphors were associated with this placement of the FUTURE in Arabic, namely KNOWING IS SEEING and RESPONSIBILITY IS BEHIND. The metaphor KNOWING IS SEEING has been found in other studies, but the metaphor RESPONSIBILITY IS BEHIND has not. As the simple meaning of the metaphor KNOWING IS SEEING is used in structuring the metaphor, the FUTURE IS BEHIND in Arabic, other components contributed to the schema of the metaphor. One of the components is the social aspect in which the first and second grammatical persons in the sentence represent the concept of people traveling along their respective JOURNEYS. When the first person wants to express a thought, they turn to face the second person, another component of the metaphor schema. This turning of the conceptual human body situates the FUTURE as being behind. Note that this turning does not need to be $180^{\circ}$ rotation but is usually partial turning. This is because the location of the second person is not necessarily behind that of the first person. The direction of the EGO in this metaphor is bi-dimensionally based. One of the dimensions is the focus dimension, and the other is the periphery. This is the focus on the destination in the JOURNEY. The front is focused on, and everything else is behind on the periphery. This focus is stated in the OED definition of the word "focus": "e. .... the point or area upon which an eye, camera, etc., is focused at a particular time." (OED Online, 25 October 2020). The conceptual metaphors that represent these concepts are FOCUS IS FRONT, and PERIPHERY IS BEHIND. This bidimensional basis matches the human focus on seeing, in which objects are either seen or unseen. As the first and second persons travel on their respective JOURNEYs, they must turn clockwise or counter-clockwise to face the other person's location to focus on that person. This turning places the FUTURE, the journey destination, on the periphery, as represented by being BEHIND in the metaphor.

The metaphors FOCUS IS FRONT and PERIPHERY IS BEHIND include the metaphor RESPONSIBILITY IS BEHIND. According to the OED, 'focus' also means ' $b$. The sustained or intense concentration of interest and attention on a particular thing ...." (OED Online, 25 October 2020). As responsibility requires sustained application of interest and attention, the lack thereof indicates a lack of focus. In other words, when interest is not sustained and attention is not paid to responsibility, there is no focus. An example of this is 19 above, in which the responsibility for bearing and raising children was expressed as being behind the first-person plural "girls". The metaphor RESPONSIBILITY is in FRONT when it is being FOCUSED on, and is BEHIND when it is in the PERIPHERY. This figurative FOCUS is an abstract concept, conceptualized mentally, and used to structure another abstract concept to indicate paying interest and attention. This is in line with Matlock et al. (2011) and Sullivan and Barth's (2012) claims about using abstract concepts to concretize other abstract concepts.

\section{CONCLUSION}

In the present study, naturally produced metaphors were analyzed. What makes this study significant is the consideration of full linguistic context, embodiment, and cultural aspects of an understudied variety of Arabic, i.e., Hijazi-Saudi Arabic. 
Arabic online tokens represent both metaphors THE FUTURE IS IN FRONT and THE FUTURE IS BEHIND. The latter of which was less productive. The metaphor THE FUTURE IS BEHIND was found associated with responsibility. Another association for placing the FUTURE behind the EGO was structuring a conceptual schema in which the EGO is turning. This turn makes the location of the FUTURE temporarily behind the EGO.

Two types of cultural specificity in metaphors were implicit in the tokens associated with the metaphor TIME IS SPACE in previous studies. One of these types is the passive availability of metaphors, henceforth available cultural specificity. The other type was when the metaphor is not accessible to other cultures therefore not used, henceforth unavailable cultural specificity. An example of the available cultural specificity is using the sun's movement to indicate the conceptual direction of the flow of time. The sun is available to all human beings; hence, it can be utilized by everyone. An example of unavailable cultural specificity is when a natural landmark is used by the people who live or have lived in a place where this landmark existed.

The metaphor pair FOCUS IS FRONT and PERIPHERY IS BEHIND is only known to be found in Arabic. Nonetheless, these metaphors' cultural specificity is available because the human body can be utilized in structuring such metaphors. The turning feature of the human body when shifting the focus from one direction to the other is another reason for this cultural specificity. Using these metaphors is not associated exclusively with culturally specific concepts that cannot be used by people of cultures other than Arabic, unlike a certain natural monument, for example. When associating time concepts with embodiment, the resulting metaphor is expected to be universal, as humans share the same experience of TIME and body peculiarities. However, once a cultural component is added, the TIME IS SPACE metaphor becomes specific to the source culture of this component.

The approach of qualitatively considering linguistic data as representations of mental conceptualization of abstract concepts has been adopted in previous research such as (Lakoff and Johnson 1999/2003, Núñez and Sweetser 2006, Sullivan and Bui 2016). In the present study, linguistic metaphors that were produced naturally in language were analyzed. Context is utilized to understand the logic behind using these metaphors. The limitation of the present study is that it does not control factors such as the age and gender of the language producers. Another limitation is that vessels of mental representations other than language, such as gestures, are not accessible when data is already written. The reason for not considering such factors and vessels is that the data analyzed was naturally produced before doing the research. This study paves the way for research on metaphors of the understudied language, i.e., Hijazi-Saudi Arabic.

\section{APPENDIX. TRANSCRIPTION CONVENTIONS}

\begin{tabular}{|c|c|c|c|}
\hline \multicolumn{4}{|c|}{ Consonants } \\
\hline Arabic Letter & Sound Symbol & Arabic Letter & Sound Symbol \\
\hline s & $?$ & 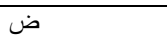 & $\mathrm{D}$ \\
\hline ب ب ب & $\mathrm{b}$ & $b$ & $\mathrm{~T}$ \\
\hline$ت$ & $\mathrm{t}$ & b & $\mathrm{Z}$ \\
\hline ث & $\theta$ & $\varepsilon$ & $\varsigma$ \\
\hline ج & 3 & $\dot{\varepsilon}$ & B \\
\hline$\tau$ & $\hbar$ & ف & $\mathrm{f}$ \\
\hline$\dot{\tau}$ & $\chi$ & ق & $q$ \\
\hline 2 & $\mathrm{~d}$ & ك & $\mathrm{k}$ \\
\hline j & ə & J & 1 \\
\hline $\mathrm{J}$ & $\mathrm{r}$ & 5 & $\mathrm{~m}$ \\
\hline$j$ & $\mathrm{z}$ & ن & $\mathrm{n}$ \\
\hline س & $\mathrm{s}$ & . & $\mathrm{h}$ \\
\hline 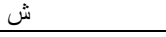 & $\int$ & 9 & $\mathrm{w}$ \\
\hline ص & $\mathrm{S}$ & ي & $\mathrm{j}$ \\
\hline
\end{tabular}

Vowel Chart
\begin{tabular}{|l|l|l|}
\hline i & & $\mathrm{u}$ \\
\hline $\mathrm{e}$ & $\curvearrowright$ & $\mathrm{o}$ \\
\hline $\mathrm{a}$ & & \\
\hline
\end{tabular}

\section{REFERENCES}

[1] Boroditsky, L. (2000). Metaphoric structuring: Understanding time through spatial metaphors. Cognition, 75(1), 1-28.

[2] Boroditsky, L., \& Ramscar, M. (2002). The roles of body and mind in abstract thought. Psychological Science, 13(2), 185-189.

[3] Boroditsky, L. (2001). Does language shape thought?: Mandarin and English speakers' conceptions of time. Cognitive psychology, 43(1), 1-22.

[4] Boroditsky, L., Fuhrman, O., \& McCormick, K. (2011). Do English and Mandarin speakers think about time differently? Cognition, 118(1), 123-129.

[5] Callizo-Romero, C., Tutnjević, S., Pandza, M., Ouellet, M., Kranjec, A., Ilić, S., Gu, Y., Göksun,T., Chahboun, S., Casasanto, D., \& Santiago, J. (2020). Temporal focus and time spatialization across cultures. Psychonomic Bulletin \& Review, 27(6), 1247-1258. 
[6] Chatterjee, A., Maher, L. M., Rothi, L. G., \& Heilman, K. M. (1995). Asyntactic thematic role assignment: The use of a temporal-spatial strategy. Brain and Language, 49(2), 125-139.

[7] Corpus of Contemporary American English, https://www.english-corpora.org/coca/ (accessed: 7/1/2020).

[8] Dahl, Ø. (1995). When the future comes from behind: Malagasy and other time concepts and some consequences for communication. International Journal of Intercultural Relations, 19(2), 197-209.

[9] De la Fuente, J., Santiago, J., Román, A., Dumitrache, C., \& Casasanto, D. (2014). When you think about it, your past is in front of you: How culture shapes spatial conceptions of time. Psychological science, 25(9), 1682-1690.

[10] Feist, M. I., \& Duffy, S. E. (2020). On the path of time: Temporal motion in typological perspective. Language and Cognition, 12(3), 444-467.

[11] Gentner, D., Imai, M., \& Boroditsky, L. (2002). As time goes by: Evidence for two systems in processing space $\rightarrow$ time metaphors. Language and cognitive processes, 17(5), 537-565.

[12] Google basic search engine, https://google.com (accessed: 2/1/2020).

[13] Kranjec, A. (2006). Extending spatial frames of reference to temporal concepts. In Forbus, K. Gentner, D., \& Regier, T. (Eds.), Proceedings of the 28thAnnual Conference of the Cognitive Science Society, 447-452. Mahwah: Lawrence Erlbaum Associates.

[14] Lakoff, G. (1993). The contemporary theory of metaphor. In A. Ortony (Ed.), Metaphor and thought (pp. 202-251).

[15] Lakoff, G., \& Johnson, M. (1980/2003). Metaphors We Live By. Chicago London: University of Chicago Press.

[16] Lakoff, G., \& Johnson, M. (1999). Philosophy in the flesh: The embodied mind and its challenge to western thought (Vol. 640). New York: Basic books.

[17] Maas, A., \& Russo, A. (2003). Directional bias in the mental representation of spatial events: nature or culture? Psychological Science, 14, 4, 296.

[18] Maher, L. M., Chatterjee, A., Rothi, L. G., \& Heilman, K. M. (1995). Agrammatic sentence production: The use of a temporalspatial strategy. Brain and Language, 49(2), 105-124.

[19] Matlock, T., Holmes, K. J., Srinivasan, M., \& Ramscar, M. (2011). Even abstract motion influences the understanding of time. Metaphor and Symbol, 26(4), 260-271.

[20] McTaggart, J. E. (1908). The unreality of time. Mind, 17(4), 457-474.

[21] Meriam-Webster dictionary, https://www.merriam-webster.com/ (accessed 7/10/2019).

[22] Núñez, R. (1999). Could the future taste purple? Reclaiming mind, body and cognition. Journal of consciousness studies, 6(1112), 41-60.

[23] Núñez, R., \& Sweetser, E. (2006). With the future behind them: Convergent evidence from Aymara language and gesture in the crosslinguistic comparison of spatial construals of time. Cognitive science, 30(3), 401-450.

[24] Núñez, R., Cooperrider, K., Doan, D., \& Wassmann, J. (2012). Contours of time: Topographic construals of past, present, and future in the Yupno valley of Papua New Guinea. cognition, 124(1), 25-35.

[25] Núñez, R.E., Motz, B.A., \& Teuscher, U. (2006). Time after time: The psychological reality of the Ego-and Time-ReferencePoint distinction in metaphorical construals of time. Metaphor and Symbol, 21, 133-146.

[26] OED Online, Oxford University Press, https://www.oed.com/viewdictionaryentry/Entry/248411 (accessed: 25/10/2020).

[27] Pragglejaz Group (2007). MIP: A method for identifying metaphorically used words in discourse. Metaphor and Symbol, 22(1), 1-39.

[28] Santiago, J., Lupáñez, J., Pérez, E., \& Funes, M. J. (2007). Time (also) flies from left to right. Psychonomic Bulletin \& Review, 14(3), 512-516.

[29] Silverman, D. (2013). Doing qualitative research: A practical handbook. Newbury Park, CA: Sage.

[30] Sullivan, J. L., \& Barth, H. C. (2012). Active (not passive) spatial imagery primes temporal judgements. Quarterly Journal of Experimental Psychology, 65(6), 1101-1109.

[31] Sullivan, K., \& Bui, L. T. (2016). With the future coming up behind them: Evidence that time approaches from behind in Vietnamese. Cognitive linguistics, 27(2), 205-233.

[32] Wierzbicka, A. (1973). In search of a semantic model of time and space. In Generative grammar in Europe (pp. 616-628). Dordrecht: Springer.

Maisarah M. Almirabi obtained his Ph.D. in Applied Linguistics at Ball State University in Muncie, Indiana, USA in 2019. He obtained his master's degree in Linguistics at the University of New Mexico in Albuquerque, New Mexico, USA in 2013. He obtained his bachelor's degree in the English Language at Umm Al-Qura University, Makkah, Saudi Arabia, in 2009.

$\mathrm{He}$ is currently an assistant professor and the Chairperson of the Department of English at Umm Al-Qura University (the main branch) in Makkah, Saudi Arabia. His research focuses on cognitive linguistics, Conceptual Metaphor Theory, pragmatics, and language documentation and revitalization. Further information can be found at: https://uqu.edu.sa/Profile/mmmirabi.

Dr. Almirabi is currently the convener of the Curriculum and Study Plan, and the Quality and Accreditation Committees in the Department of English and a member on the Curriculum and Study Plan Committee in the College of Social Sciences at Umm AlQura University, Makkah, Saudi Arabia. 